\title{
Dopamine sensing based on ultrathin fluorescent metal-organic nanosheets
}

\author{
Faezeh Moghzi, ${ }^{a}$ Janet Soleimannejad ${ }^{a}$, Eva Carolina Sañudo ${ }^{b}, J_{a n}$ Janczak $^{c}$ \\ ${ }^{a}$ School of Chemistry, College of Science, University of Tehran, P.O. Box 14155-6455, Tehran, Iran \\ ${ }^{b}$ Departament de Química Inorgànica i Institut de Nanociència i Nanotecnologia, Universitat de Barcelona. Av. \\ Diagonal 645, 08028 Barcelona, Spain \\ 'Institute of Low Temperature and Structure Research, Polish Academy of Science, P.O. Box 1410, 50950, Wroclaw, \\ Poland.
}

\section{Contents}

SI 1. Reagents and Instruments

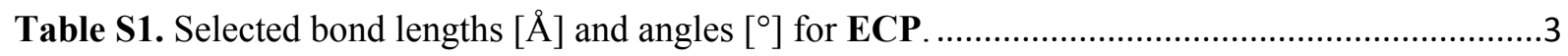

Table S2. Hydrogen bonds' geometry $\left(\AA,^{\circ}\right)$ of ECP.

Figure S1. Results of 5, 10, 20, 30 and $60 \mathrm{~min}$ of sonication of dispersions of ECP in ethanol, acetone and water.

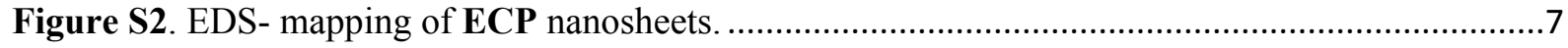

Figure S3. Absorption spectra of the $\mathrm{H}_{2}$ pzdc organic ligand and dopamine in distilled water..............7

Figure S4. The emission intensity of bulk crystals and nanoflakes of ECP..................................

Figure S5. Fluorescence spectra of ECP nanoflakes in presence of dopamine at different points of time upon excitation at $280 \mathrm{~nm}$.

Figure S6. Corresponding luminescence intensity changes as a function of dopamine concentration upon excitation at $280 \mathrm{~nm}$.

Figure S7. DA sensing efficiency of ECP nanoflaks during four cycles of DA adsorption-desorption.

Figure S8. Luminescence decay curves of $\mathrm{Eu}^{3+}$ emission at $280 \mathrm{~nm}$ in ECP nanosheets with and without the addition of dopamine $(10 \mu \mathrm{M})$....

Table S3. Comparison of the performance of dopamine optical sensing in previous and present studies. 10

Reference

\footnotetext{
${ }^{*}$ Corresponding Author

J. Soleimannejad, email; janet_soleimannejad@khayam.ut.ac.ir
} 


\section{SI 1. Reagents and Instruments}

Reagents used in this work were supplied from Sigma-Aldrich and consumed without more purification. Solvents including ethanol and acetone were bought from Scharlab.

Ultrasound was applied with a Branson Digital Sonifier SFX 550 (Emerson) sonicator with maximum power 550W. PXRD patterns were recorded on PANalytical X'Pert PRO MRD equipped with a $\mathrm{CuK} \alpha$ radiation source $(\lambda=1.54184 \AA$ ). SEM images of platinum-metallized ECP nanoflakes were obtained with a FEI Quanta 650F microscope with a beam voltage at 20 $\mathrm{kV}$. Nanoflakes suspension were deposited onto aluminum substrate by drop-casting method. Energy Dispersive X-ray Spectroscopy (EDS) was coupled with FEI Quanta 650F microscope. FT-IR spectra of ECP were measured by utilizing a Tensor 27 (Bruker) spectrophotometer with $\mathrm{KBr}$ pellet in the range of $400-4000 \mathrm{~cm}^{-1}$. STEM images were collected on a Magellan $400 \mathrm{~L}$ from FEI operated at $20 \mathrm{kV}$ and 100 pA. Both Bright Field (BF) and High-Angle Annular Dark Field (HAADF) detectors were used simultaneously. The samples were prepared by dropcasting of suspension on top of STEM grid (ultrathin carbon type-A, 400 mesh, copper, approx. grid hole size $42 \mu \mathrm{m}$ ) and solvent evaporate in the room temperature. Thermogravimetric analysis was performed on a Perkin Elmer thermogravimetric analyzer from room temperature to $1000{ }^{\circ} \mathrm{C}$ with a heating rate of $10 \mathrm{~K} / \mathrm{min}$ under $\mathrm{N}_{2}$ atmosphere. Optical and fluorescence microscopy images were obtained using a Zeiss Axio Observer Z1m inverted optical microscope. Absorption spectra of the organic solutions and water suspensions were registered in the Agilent Cary 60 spectrophotometer using a 1x1 cm quartz cuvette. Solid samples were measured utilizing an integrating sphere (Agilent Cary 60 remote diffuse reflectance accessory). The Kubelka-Munk equation $\left(\mathrm{F}(\mathrm{R})=(1-\mathrm{R})^{2} / 2 \mathrm{R}\right)$ was used to estimate the absorption spectra from the experimental diffused reflectance. Emission spectra of ECP nanoflakes were recorded through the PTI QuantaMaster 300 phosphorescence/fluorescence spectrophotometer (Horiba Ltd.). Surface topography imaging of ECP nanoflakes carried out in tapping mode using beam shaped silicon cantilevers (Nanosensors, nominal force constant: $5 \mathrm{~N} \cdot \mathrm{m}-1$, tip radius: $\sim 7 \mathrm{~nm}$ ) on an Agilent 5500 AFM/SPM microscope.

Colorless single crystal of ECP was selected to collect data on a four-circle KUMA KM4 diffractometer with a two-dimensional CCD area detector. The graphite monochromatized MoK $\alpha$ radiation $(\lambda=0.71073 \AA)$ and the $\omega$-scan technique $\left(\Delta \omega=1^{\circ}\right)$ were applied for data collection. Data collection and reduction, along with absorption correction, were accomplished via the CrysAlis software package [1]. The structure was solved by the direct methods using SHELXT [2] giving positions of almost all non-hydrogen atoms. The other atoms were 
localized with subsequent difference Fourier syntheses. The structure was refined using SHELXL-2018 [2] with the anisotropic thermal displacement parameters. The hydrogen atoms of the aromatic ring were refined with the riding model, and $\mathrm{H}$ atoms of water molecules were constrained with $\mathrm{U}_{\text {iso }}(\mathrm{H})=1.5 \mathrm{U}_{\mathrm{eq}}(\mathrm{O})$ and $\mathrm{O}-\mathrm{H}=0.87 \AA$.

Table S1. Selected bond lengths $[\AA]$ and angles $\left[{ }^{\circ}\right]$ for ECP.

\begin{tabular}{|c|c|c|c|}
\hline \multicolumn{4}{|l|}{ Bond lengths $(\AA)$} \\
\hline Eu-O1 & $2.358(3)$ & $\mathrm{Eu}-\mathrm{O} 2 \mathrm{~W}$ & $2.430(3)$ \\
\hline $\mathbf{E u}-\mathbf{O 1 4 ^ { \mathrm { i } }}$ & $2.359(3)$ & $\mathrm{Eu}-\mathrm{O} 1 \mathrm{~W}$ & $2.534(3)$ \\
\hline $\mathbf{E u}-\mathbf{O} 4^{\mathrm{ii}}$ & $2.387(4)$ & $\mathrm{Eu}-\mathrm{N} 4^{\mathrm{ii}}$ & $2.735(4)$ \\
\hline $\mathbf{E u}-011$ & $2.391(3)$ & $\mathrm{Eu}-\mathrm{N} 14^{\mathrm{i}}$ & $2.778(4)$ \\
\hline $\mathbf{E u}-\mathbf{O 3 W}$ & $2.429(3)$ & & \\
\hline \multicolumn{4}{|l|}{ Bond angles ( ${ }^{\circ}$ ) } \\
\hline O1-Eu-O14 & $79.63(11)$ & $\mathrm{O} 2 \mathrm{~W}-\mathrm{Eu}-\mathrm{N} 4^{\mathrm{ii}}$ & $73.39(11)$ \\
\hline O1-Eu-O4 & 87.27 (12) & $\mathrm{O} 1 \mathrm{~W}-\mathrm{Eu}-\mathrm{N} 4^{\mathrm{ii}}$ & $116.41(12)$ \\
\hline $014^{\mathrm{i}}-\mathbf{E u}-O 4^{\mathrm{ii}}$ & $129.36(12)$ & $\mathrm{O} 1-\mathrm{Eu}-\mathrm{N} 14^{\mathrm{i}}$ & $128.72(12)$ \\
\hline O1-Eu-O11 & $77.42(12)$ & $\mathrm{O} 14^{\mathrm{i}}-\mathrm{Eu}-\mathrm{N} 14^{\mathrm{i}}$ & $61.79(12)$ \\
\hline O14 $-\mathbf{E u}-011$ & $82.38(12)$ & $\mathrm{O} 4^{\mathrm{ii}}-\mathrm{Eu}-\mathrm{N} 14^{\mathrm{i}}$ & $143.13(11)$ \\
\hline O4i $-\mathbf{E u}-011$ & $141.77(11)$ & $\mathrm{O} 11-\mathrm{Eu}-\mathrm{N} 14^{\mathrm{i}}$ & $65.76(11)$ \\
\hline O1-Eu-O3W & $139.37(11)$ & $\mathrm{O} 3 \mathrm{~W}-\mathrm{Eu}-\mathrm{N} 14^{\mathrm{i}}$ & $73.78(12)$ \\
\hline O14 $-\mathbf{E u}-03 \mathrm{~W}$ & $134.99(12)$ & $\mathrm{O} 2 \mathrm{~W}-\mathrm{Eu}-\mathrm{N} 14^{\mathrm{i}}$ & $68.17(12)$ \\
\hline $\mathbf{O} 4^{i i}-\mathbf{E u}-\mathbf{O 3 W}$ & $83.17(12)$ & $\mathrm{O} 1 \mathrm{~W}-\mathrm{Eu}-\mathrm{N} 14^{\mathrm{i}}$ & $123.69(12)$ \\
\hline O11-Eu-O3W & $86.14(11)$ & $\mathrm{N} 4^{\mathrm{ii}}-\mathrm{Eu}-\mathrm{N} 14^{\mathrm{i}}$ & $119.79(12)$ \\
\hline O1-Eu-O2W & $140.35(11)$ & $\mathrm{O} 2 \mathrm{~W}-\mathrm{Eu}-\mathrm{O} 1 \mathrm{~W}$ & $134.42(11)$ \\
\hline O14 $-\mathrm{Eu}-\mathrm{O} 2 \mathrm{~W}$ & $81.25(11)$ & $\mathrm{O} 1-\mathrm{Eu}-\mathrm{N} 4^{\mathrm{ii}}$ & $67.31(11)$ \\
\hline $\mathbf{O} 4^{\mathrm{ii}}-\mathbf{E} \mathbf{u}-\mathbf{O} 2 \mathrm{~W}$ & $78.60(12)$ & $\mathrm{O} 14^{\mathrm{i}}-\mathrm{Eu}-\mathrm{N} 4^{\mathrm{ii}}$ & $68.12(13)$ \\
\hline O11-Eu-O2W & $133.56(12)$ & $\mathrm{O} 4^{\mathrm{ii}}-\mathrm{Eu}-\mathrm{N} 4^{\mathrm{ii}}$ & $61.71(12)$ \\
\hline $\mathrm{O} 3 \mathrm{~W}-\mathrm{Eu}-\mathrm{O} 2 \mathrm{~W}$ & $75.79(11)$ & $\mathrm{O} 11-\mathrm{Eu}-\mathrm{N} 4^{\mathrm{ii}}$ & $137.00(11)$ \\
\hline O1-Eu-O1W & $71.33(11)$ & $\mathrm{O} 3 \mathrm{~W}-\mathrm{Eu}-\mathrm{N} 4^{\mathrm{ii}}$ & $136.81(12)$ \\
\hline $011-\mathbf{E u}-01 \mathrm{~W}$ & $71.58(11)$ & $\mathrm{O} 11-\mathrm{Eu}-\mathrm{O} 1 \mathrm{~W}$ & $71.58(11)$ \\
\hline O3W-Eu-O1W & $68.25(11)$ & $\mathrm{O} 3 \mathrm{~W}-\mathrm{Eu}-\mathrm{O} 1 \mathrm{~W}$ & $68.25(11)$ \\
\hline
\end{tabular}

Symmetry codes: (i) $-x+1,-y+1,-z+1$; (ii) $x+1 / 2, y,-z+1 / 2$. 
Table S2. Hydrogen bonds' geometry $\left(\AA,^{\circ}\right)$ of ECP.

\begin{tabular}{lllll}
\hline$D-\mathrm{H} \cdots A$ & $D-\mathrm{H}$ & $\mathrm{H} \cdots A$ & $D \cdots A$ & $D-\mathrm{H} \cdots A$ \\
\hline $\mathrm{N} 11-\mathrm{H} 11 \cdots \mathrm{O} 2$ & 0.88 & 1.73 & $2.605(6)$ & 172 \\
$\mathrm{O} 1 W-\mathrm{H} 1 W B \cdots \mathrm{O} 3$ & 0.88 & 1.98 & $2.853(5)$ & 178 \\
$\mathrm{~N} 11-\mathrm{H} 11 \cdots \mathrm{O} 2$ & 0.88 & 1.73 & $2.605(6)$ & 172 \\
$\mathrm{O} 1 W-\mathrm{H} 1 W B \cdots \mathrm{O} 3$ & 0.88 & 1.98 & $2.853(5)$ & 178 \\
$\mathrm{O} 2 W-\mathrm{H} 2 W A \cdots \mathrm{N} 14^{\mathrm{i}}$ & 0.87 & 2.44 & $2.933(6)$ & 116 \\
$\mathrm{O} 2 W-\mathrm{H} 2 W A \cdots \mathrm{O} 12^{\mathrm{ii}}$ & 0.87 & 1.98 & $2.784(5)$ & 154 \\
$\mathrm{O} 2 W-\mathrm{H} 2 W B \cdots \mathrm{O} 3^{\mathrm{ii}}$ & 0.87 & 2.23 & $2.717(5)$ & 115 \\
$\mathrm{O} 3 W-\mathrm{H} 3 W A \cdots \mathrm{O} 2^{\mathrm{iii}}$ & 0.87 & 2.14 & $2.730(4)$ & 125 \\
$\mathrm{O} 3 W-\mathrm{H} 3 W B \cdots \mathrm{O} 13^{\mathrm{iv}}$ & 0.87 & 2.26 & $2.721(5)$ & 113 \\
\hline Symmetry codes: (i) $-x+1,-y+1,-z+1 ;($ ii $)$ & $x+1, y, z ;($ iii) $-x+1 / 2, y+1 / 2, z ;(\mathrm{iv}) x+1 / 2,-y+3 / 2,-z+1$.
\end{tabular}



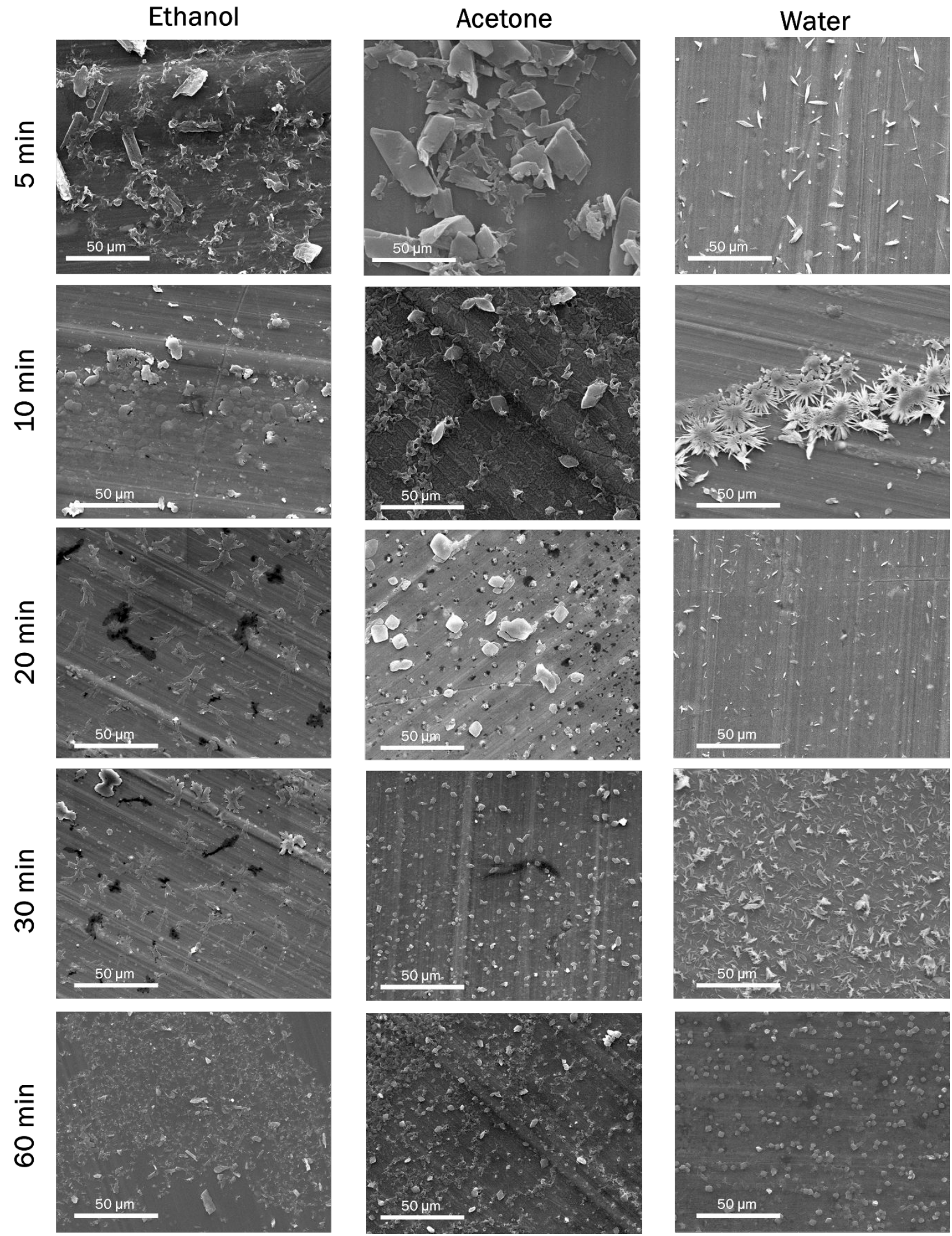

Figure S1. Results of 5, 10, 20, 30 and 60 min of sonication of dispersions of ECP in ethanol, acetone and water. 

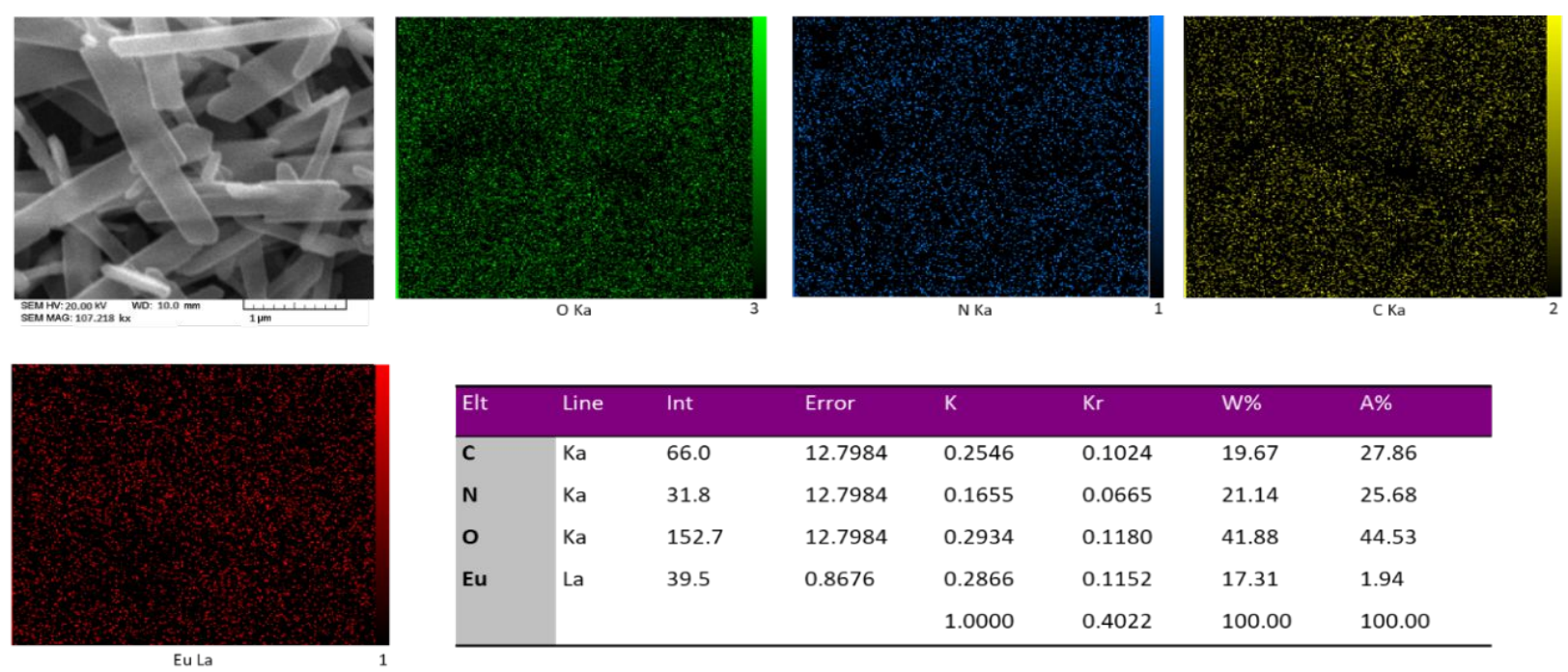

\begin{tabular}{llllllll}
\hline Elt & Line & Int & Error & K & Kr & W\% & A\% \\
\hline $\mathrm{C}$ & Ka & 66.0 & 12.7984 & 0.2546 & 0.1024 & 19.67 & 27.86 \\
$\mathrm{~N}$ & $\mathrm{Ka}$ & 31.8 & 12.7984 & 0.1655 & 0.0665 & 21.14 & 25.68 \\
$\mathrm{O}$ & $\mathrm{Ka}$ & 152.7 & 12.7984 & 0.2934 & 0.1180 & 41.88 & 44.53 \\
Eu & La & 39.5 & 0.8676 & 0.2866 & 0.1152 & 17.31 & 1.94 \\
& & & & 1.0000 & 0.4022 & 100.00 & 100.00 \\
\hline
\end{tabular}

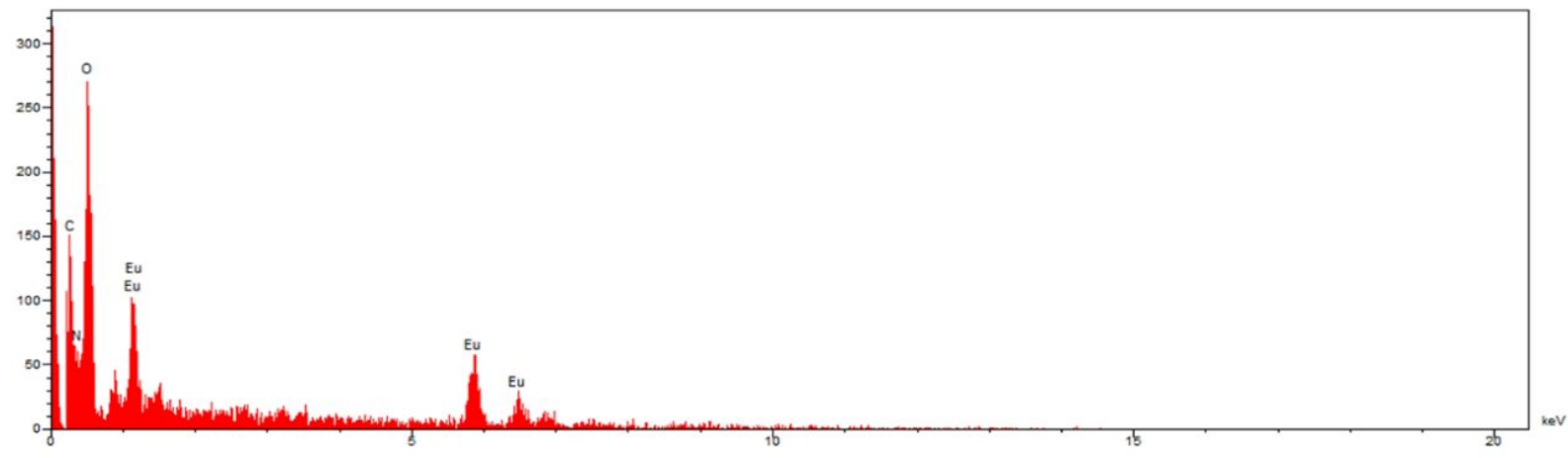

Figure S2. EDS- mapping of ECP nanosheets.
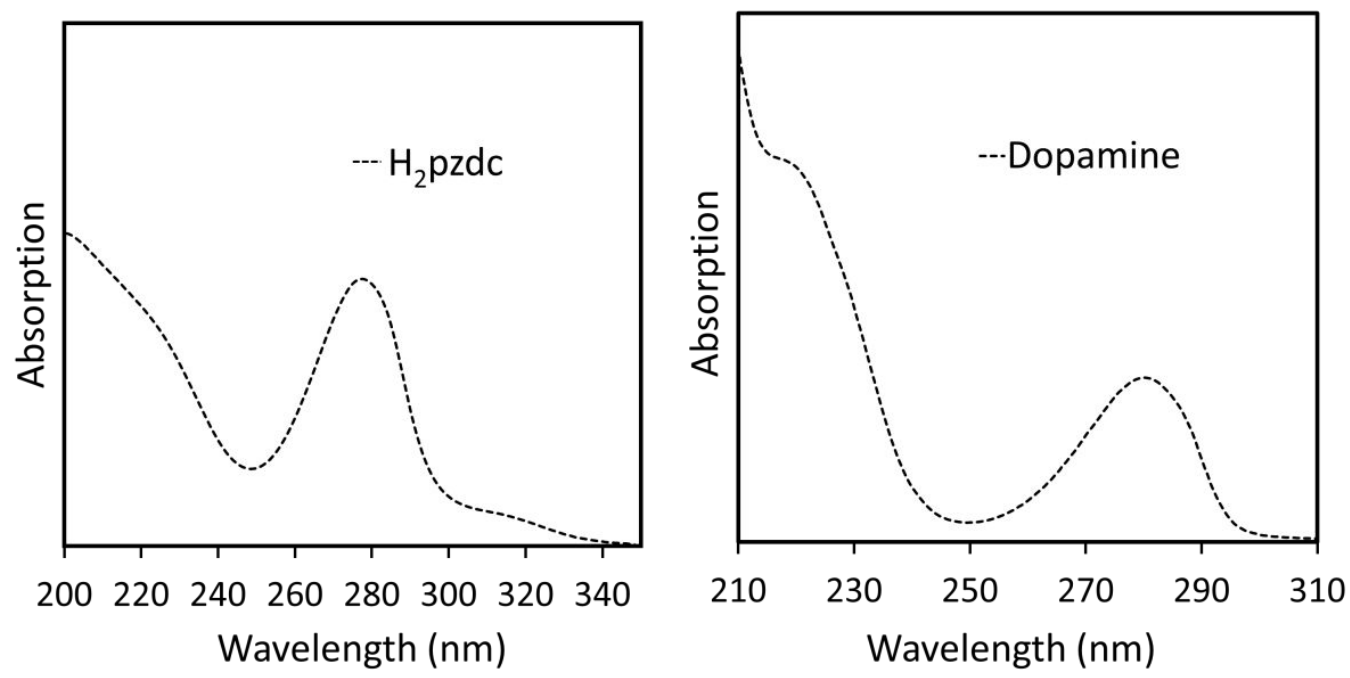

Figure S3. Absorption spectra of the $\mathrm{H}_{2}$ pzdc organic ligand and dopamine in distilled water. 


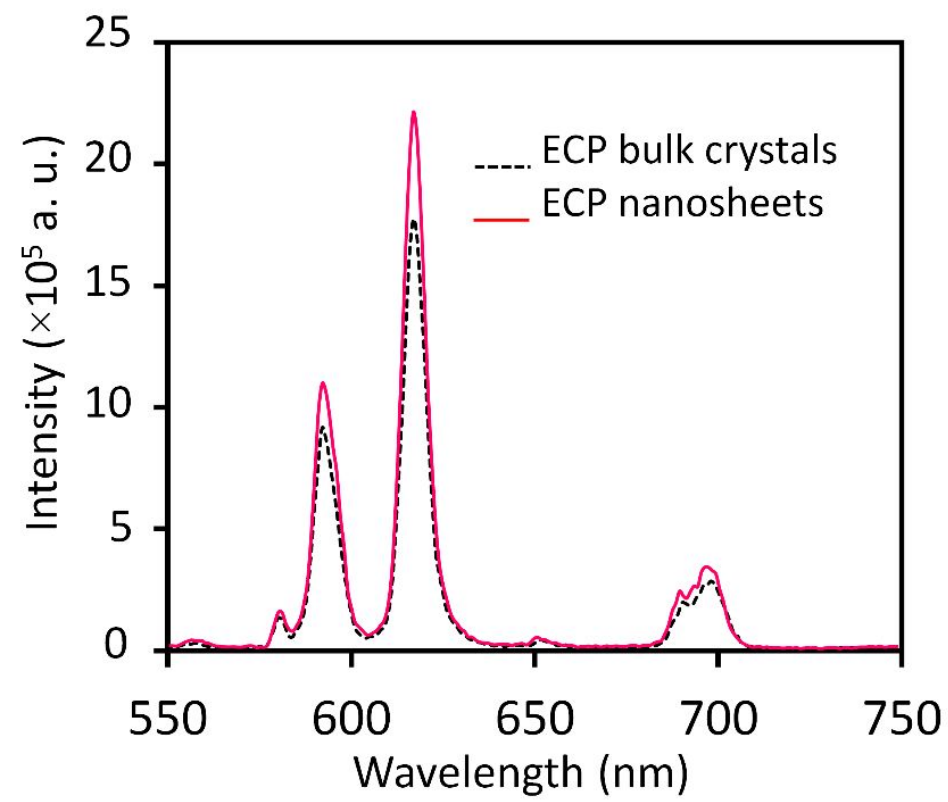

Figure S4. The emission intensity of bulk crystals and nanoflakes of ECP.

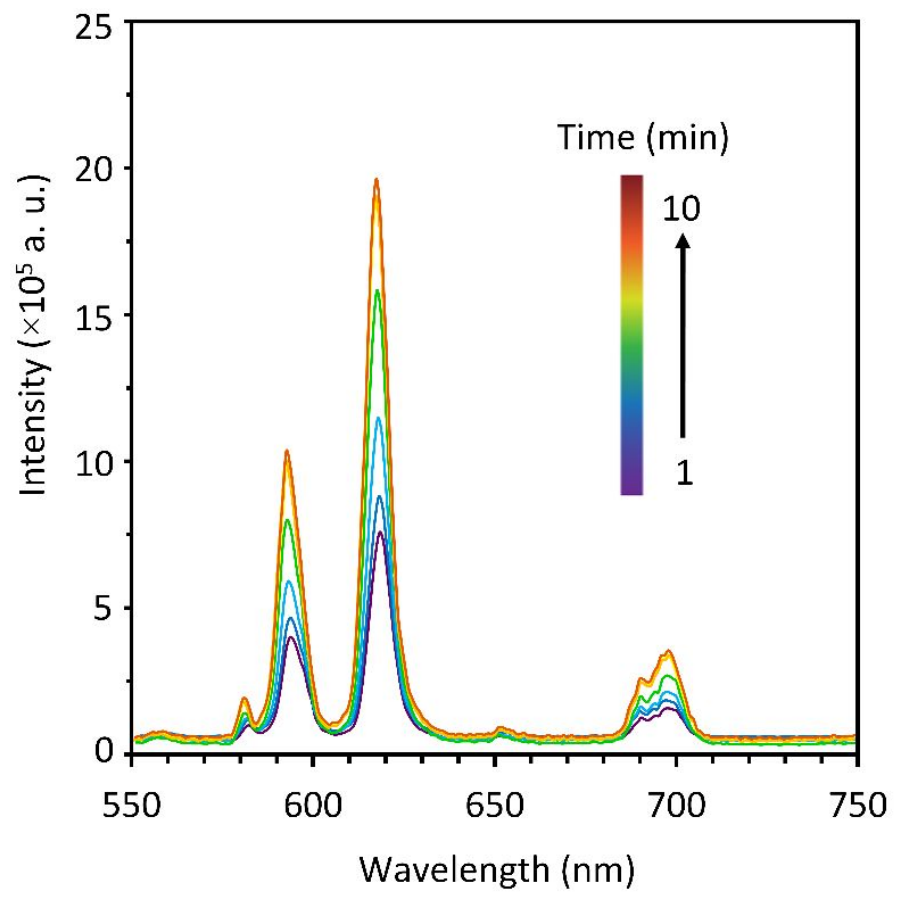

Figure S5. Fluorescence spectra of ECP nanoflakes in presence of dopamine at different points of time upon excitation at $280 \mathrm{~nm}$. 


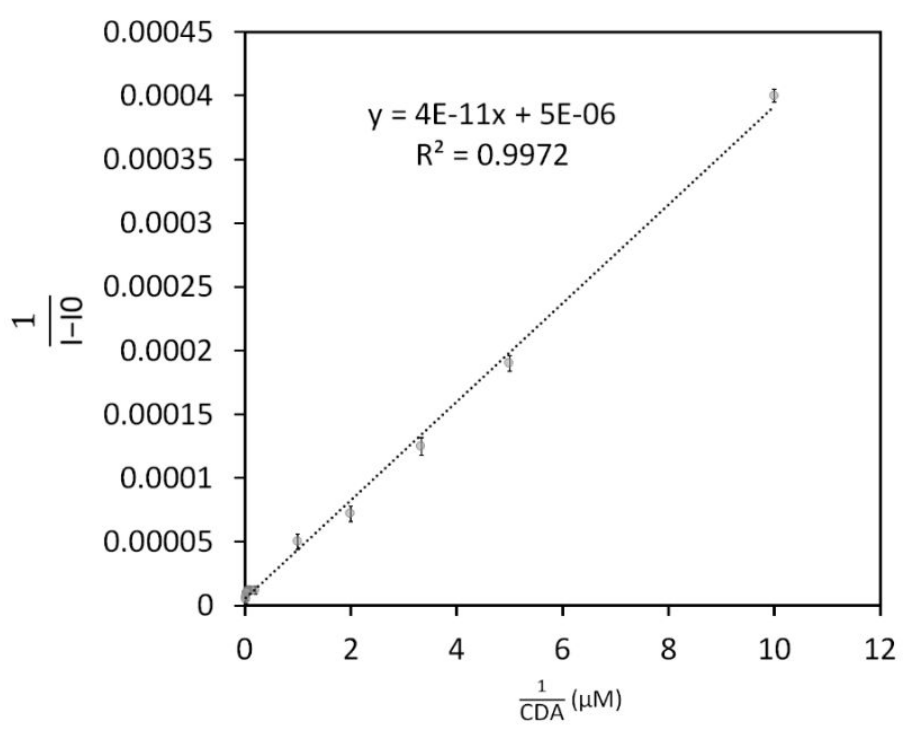

Figure S6. Corresponding luminescence intensity changes as a function of dopamine concentration upon excitation at $280 \mathrm{~nm}$.

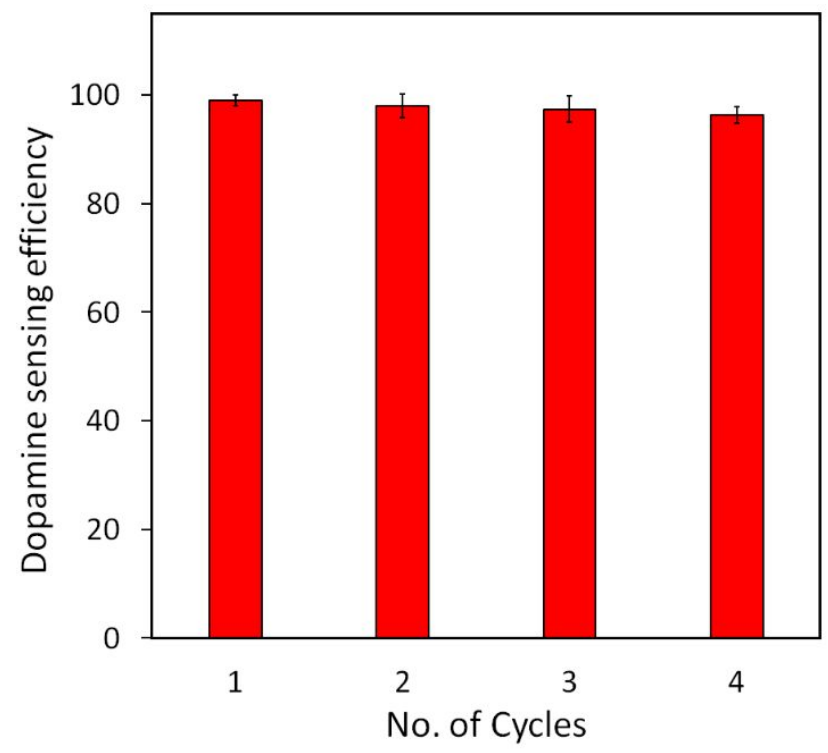

Figure S7. DA sensing efficiency of ECP nanoflaks during four cycles of DA adsorptiondesorption. 


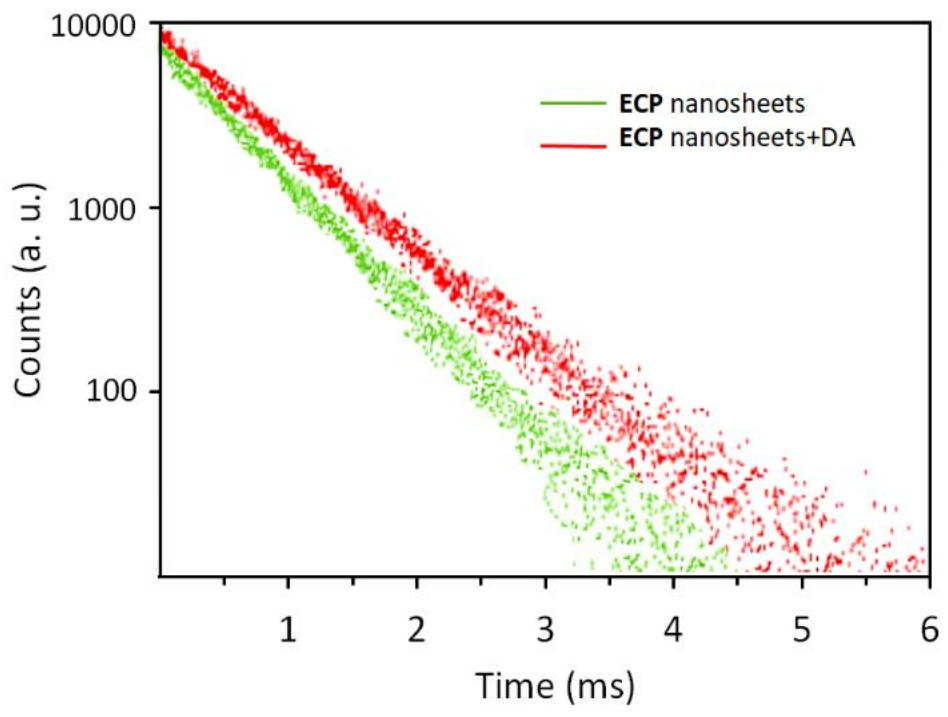

Figure S8. Luminescence decay curves of $\mathrm{Eu}^{3+}$ emission at $280 \mathrm{~nm}$ in $\mathbf{E C P}$ nanosheets with and without the addition of dopamine $(10 \mu \mathrm{M})$.

Table S3. Comparison of the performance of dopamine optical sensing in previous and present studies.

\begin{tabular}{lccccc}
\hline Materials & $\begin{array}{c}\text { Time required } \\
(\mathbf{m i n})\end{array}$ & $\begin{array}{c}\text { Detection limit } \\
(\mathbf{n M})\end{array}$ & Sample & $\begin{array}{c}\text { Recovery } \\
(\%)\end{array}$ & Reference \\
\hline $\mathrm{Abtz}_{-} \mathrm{CdI}_{2}-\mathrm{MOF}$ & 50 & 57 & Urine & $94.5-102$ & 3 \\
$\mathrm{NaGdF}_{4}$ :Tb nanoparticles & 5 & 30 & Human serum & $89-100$ & 4 \\
Graphene oxide & 10 & 94 & Human serum & $102-110$ & 5 \\
Polydopamine nanoparticles & 30 & 40 & - & - & 6 \\
Gold nanoclusters & 90 & 1 & Real sample & $93.5-108$ & 7 \\
$\mathrm{Silicon}$ nanoparticles & 180 & 0.3 & - & - & 8 \\
$\mathrm{Fe}_{3} \mathrm{O}_{4} @ \mathrm{SiO}_{2}$-NH-EDTA- & - & 820 & Human serum & $97-101$ & 9 \\
$\mathrm{~Tb}(\mathrm{III})$ & & & & & \\
$\mathrm{C} 2-\mathrm{F} 127$ organic nanoparticles & 90 & 35 & - & - & 10 \\
ECP nanoflakes & 5 & 21 & Human serum & $90-99$ & This work \\
\hline
\end{tabular}




\section{Reference}

1. CrysAlis CCD and CrysAlis Red 1.171.38.43, Rigaku Oxford Diffraction, 2015.

2. G. M. Sheldrick, Crystal structure refinement with SHELXL. Acta Crystallographica. C, 7(2015) 3-8.

3. Cheng, Y; Wu, J; Guo, Ch; Li, X-G; Ding, B; Li, Y; A facile water-stable MOF-based "off on" fluorescent switch forlabel-free detection of dopamine in biological fluid. J. Mater. Chem. B, 2017,5, 2524-2535.

4. Ling, X; Shi, R; Zhang, J; Liu, D; Weng, M; Zhang, Ch-W; LU, M; Xie, X; Huang, L; Huang, W; Dual-Signal Luminescent Detection of Dopamine by a Single Type of Lanthanide Doped Nanoparticles. ACS Sens. 2018, 3, 9, 1683-1689.

5. Chen, J.; Yan, X.; Meng, K.; Wang, S. Graphene Oxide based Photoinduced Charge Transfer LabelFree Near-Infrared Fluorescent Biosensor for Dopamine. Anal. Chem. 2011,83, 8787-8793.

6. Yildirim, A.; Bayindir, M. Turn-on Fluorescent Dopamine Sensing Based on in Situ Formation of Visible Light Emitting Polydopamine Nanoparticles. Anal. Chem. 2014, 86, 5508-5512.

7. Teng, Y.; Jia, X.; Li, J.; Wang, E. Ratiometric Fluorescence Detection of Tyrosinase Activity and Dopamine Using Thiolate-Protected Gold Nanoclusters. Anal. Chem. 2015, 87, 4897-4902.

8. Zhang, X.; Chen, X.; Kai, S.; Wang, H.; Yang, J.; Wu, F.; Chen, Z. Highly Sensitive and Selective Detection of Dopamine using One-Pot Synthesized Highly Photoluminescent Silicon Nanoparticles. Anal. Chem. 2015, 87, 3360-3365.

9. Chen, M., Zheng, Y.; Gao, J.; Li, C.; Yu, C.; Wang, Q. Fluorometric Determination of Dopamine by Using a Terbium (III) Inorganic-Organic Network. Microchimica Acta 2017, 184, 2275-2280.

10. Ding, L.; Qin, Z.; Xiang, C.; Zhou, G. Novel Fluorescent Organic Nanoparticles as a Label-Free Biosensor for Dopamine in Serum. J. Mater. Chem. B 2017, 5, 2750-2756. 